\title{
Synthesis, Characterization of Substituted Piperdin-4-ones with Dichloro(cyclooctadiene)palladium(II)
}

\author{
NALINI NAMASIVAYAM and CHINNIAGOUNDER THEIVARASU* \\ Department of Chemistry, PSG College of Technology, Coimbatore 641004, India \\ theivarasu@yahoo.co.in
}

Received 26 September 2013 / Accepted 8 November 2013

\begin{abstract}
The complexes were synthesized using substituted 2,6-diphenyl piperdin-4-ones (ligands) and dichloro(cyclooctadiene) palladium(II) (precursor) in dichloromethane (solvent). In the present study we report the synthesis and characterized by infrared and NMR studies on substituted 2,6-diphenylpiperdin-4-ones and their palladium(II)complex.
\end{abstract}

Keywords: Synthesis, 2,6-Diphenyl piperdin-4-ones, Precursor, Dichloro(cyclooctadiene) palladium(II)

\section{Introduction}

4-Piperidone play an important role which embraces the organometallic field in different ascepts $^{1-5}$. 2,6-Diphenyl-4-piperidone and their metal complexes have a variety of applications in numerous scientific areas. By exhibiting a wide range of pharmacological activities $^{6-12}$ a series of 2,6-diphenyl-4-piperidone were synthesized by different dialkyl ketone, aromatic aldehyde and ammonium acetate by Mannich condensation reaction ${ }^{13}$. The formation of an interesting array of new compounds we reported the synthesis of substituted 2, 6-diphenyl-4-piperidone with palladium metal by the formation of an interesting array of new compounds. These palladium (II) complexes have gained more significant applications.

\section{Experimental}

The reagent grade chemicals and reagents were purchased from AR grade and purified by either distillation or recrystallization before use. The purity of the synthesized compounds was checked by thin layer chromatography (TLC) with silica gel plates.

\section{Physical measurements}

Melting points of the synthesized compounds were taken in open glass capillaries using a Barnstead 9001 electro thermal melting point apparatus and are uncorrected. Infrared (IR) spectra $\left(v, \mathrm{~cm}^{-1}\right)$ were recorded on a Shimadzu FT/IR-330E, Fourier Transform Infrared Spectrometer using $\mathrm{KBr}$ discs. Nuclear Magnetic spectroscopic (NMR) measurements ${ }^{1} \mathrm{H}$ NMR and ${ }^{13} \mathrm{C}$ NMR spectra were noted by Jeol GSX 4OONB NMR spectrometer operating at $500.13 \mathrm{MHz}$ and 125.76 MHz with $\mathrm{DMSOd}_{6}$ as solvent and tetramethylsilane (TMS) as an internal standard. 


\section{Synthesis of substituted 2, 6-diaryl piperidin-4-ones $\left(L_{1}-L_{9}\right)$}

The general procedure for the preparation of substituted piperdin-4-ones is reported by literature method ${ }^{13}$. The ligands $\left(\mathrm{L}_{1}-\mathrm{L}_{9}\right)$ were prepared by refluxing a mixture of substituted aldehydes, dry ammonium acetate and dialkyl ketones $(2: 1: 1)$ in the presence of ethanol $(30 \mathrm{~mL})$. This reaction mixture was allowed to stand overnight at room temperature followed by adding concentrated hydrochloric acid $(30 \mathrm{~mL})$. Then the precipitated hydrochloride was collected and washed with ethanol and ether mixture (1:5) and it was transferred to one litre beaker. The hydrochloride was suspended in acetone and basified with a strong ammonia solution. On dilution with excess of water the free base was separated out. The product was filtered, washed with water and dried. Crystallization of the product from ethanol results in substituting piperidin-4-ones. The structure of substituted piperidin-4-ones ligands as shown in Figure1.<smiles>[R]C1NC([R])C([R])C(=O)C1[R]</smiles>

Figure 1. Structure of substituted piperidin-4-ones ligands

\begin{tabular}{cccccc}
\hline Ligand & Ligand name & $\mathrm{R}_{1}$ & $\mathrm{R}_{2}$ & $\mathrm{R}_{3}$ & $\mathrm{R}_{4}$ \\
\hline $\mathrm{L}_{2}$ & 3-Methyl-2,6-diphenylpiperidin-4-one & $\mathrm{C}_{6} \mathrm{H}_{5}$ & $\mathrm{C}_{6} \mathrm{H}_{5}$ & $\mathrm{CH}_{3}$ & $\mathrm{H}$ \\
$\mathrm{L}_{2}$ & 3,5-Dimethyl-2,6-diphenylpiperin-4-one & $\mathrm{C}_{6} \mathrm{H}_{5}$ & $\mathrm{C}_{6} \mathrm{H}_{5}$ & $\mathrm{CH}_{3}$ & $\mathrm{CH}_{3}$ \\
$\mathrm{~L}_{3}$ & 3-Ethyl-2,6-diphenylpieridine-4-one & $\mathrm{C}_{6} \mathrm{H}_{5}$ & $\mathrm{C}_{6} \mathrm{H}_{5}$ & $\mathrm{C}_{2} \mathrm{H}_{5}$ & $\mathrm{H}$ \\
$\mathrm{L}_{4}$ & 3-Methyl-2,6-ditolylpiperidin-4-one & $\mathrm{C}_{6} \mathrm{H}_{4} \mathrm{CH}_{3}$ & $\mathrm{C}_{6} \mathrm{H}_{4} \mathrm{CH}_{3}$ & $\mathrm{CH}_{3}$ & $\mathrm{H}$ \\
$\mathrm{L}_{5}$ & 3,5-Dimethyl-2,6- ditolylpiperidin-4-one & $\mathrm{C}_{6} \mathrm{H}_{4} \mathrm{CH}_{3}$ & $\mathrm{C}_{6} \mathrm{H}_{4} \mathrm{CH}_{3}$ & $\mathrm{CH}_{3}$ & $\mathrm{CH}_{3}$ \\
$\mathrm{~L}_{6}$ & 3-Ethyl-2,6- ditolylpiperidin-4-one & $\mathrm{C}_{6} \mathrm{H}_{4} \mathrm{CH}_{3}$ & $\mathrm{C}_{6} \mathrm{H}_{4} \mathrm{CH}_{3}$ & $\mathrm{C}_{2} \mathrm{H}_{5}$ & $\mathrm{H}$ \\
$\mathrm{L}_{7}$ & 3-Methyl-2,6-dianisylpiperidin-4-one & $\mathrm{C}_{6} \mathrm{H}_{4} \mathrm{OCH}_{3}$ & $\mathrm{C}_{6} \mathrm{H}_{4} \mathrm{OCH}_{3}$ & $\mathrm{CH}_{3}$ & $\mathrm{H}$ \\
$\mathrm{L}_{8}$ & 3,5-Dimethyl-2,6- dianisylpiperidin-4-one & $\mathrm{C}_{6} \mathrm{H}_{4} \mathrm{OCCH}_{3}$ & $\mathrm{C}_{6} \mathrm{H}_{4} \mathrm{OCH}_{3}$ & $\mathrm{CH}_{3}$ & $\mathrm{CH}_{3}$ \\
$\mathrm{~L}_{9}$ & 3-Ethyl-2,6- dianisylpiperidin-4-one & $\mathrm{C}_{6} \mathrm{H}_{4} \mathrm{OCH}_{3}$ & $\mathrm{C}_{6} \mathrm{H}_{4} \mathrm{OCH}_{3}$ & $\mathrm{C}_{2} \mathrm{H}_{5}$ & $\mathrm{H}$ \\
\hline
\end{tabular}

Preparation of palladium(II) ion complexes $\left(C_{1-} C_{9}\right)$

The dichloro(cyclooctadiene)palladium (II) is used as a precursor. The dichloro (cyclooctadiene) palladium(II) with 2, 6-diaryl piperidin-4-ones ligands $\left(\mathrm{L}_{1}-\mathrm{L}_{9}\right)$ were prepared by the following procedure: A mixture of dichloro(cyclooctadiene) palladium(II) $(0.5 \mathrm{~m} \mathrm{~mol})$ and substituted piperidin-4-ones $\left(\mathrm{L}_{1}-\mathrm{L}_{9}\right)(0.5 \mathrm{~m} \mathrm{~mol}$ in dichloromethane $(50 \mathrm{~mL})$ was refluxed for $5 \mathrm{~h}$. The solvent was then distilled off under reduced pressure. The residue was repeatedly washed with hot ethanol, acetone and ether remove the unreacted piperidin-4-one and then dried through in vacuo phosphorus (V) oxide.

\section{Results and Discussion}

The general schematic representation describing the routes of synthesis was furnished in Figure 2. By the condensation of substituted aldehyde, ammonium acetate and dialkyl ketones in the ratio of 2:1:1 for obtaining substituted 2, 6-diarylpiperdin-4-ones. All the synthesized substituted 2, 6-diarylpiperdin-4-ones are soluble in solvents such as ethanol, methanol, dimethylsulphoxide, dichloromethane and ether but insoluble in water. They are stable in air under dry conditions. Palladium(II) complexes are dull yellowish in colour. The structure of the synthesized substituted 2, 6-diarylpiperdin-4-ones is established on the basis of IR and NMR $\left({ }^{1} \mathrm{H}\right.$ and $\left.{ }^{13} \mathrm{C}\right)$ data obtained as given in Table 1,2 and 3 . 

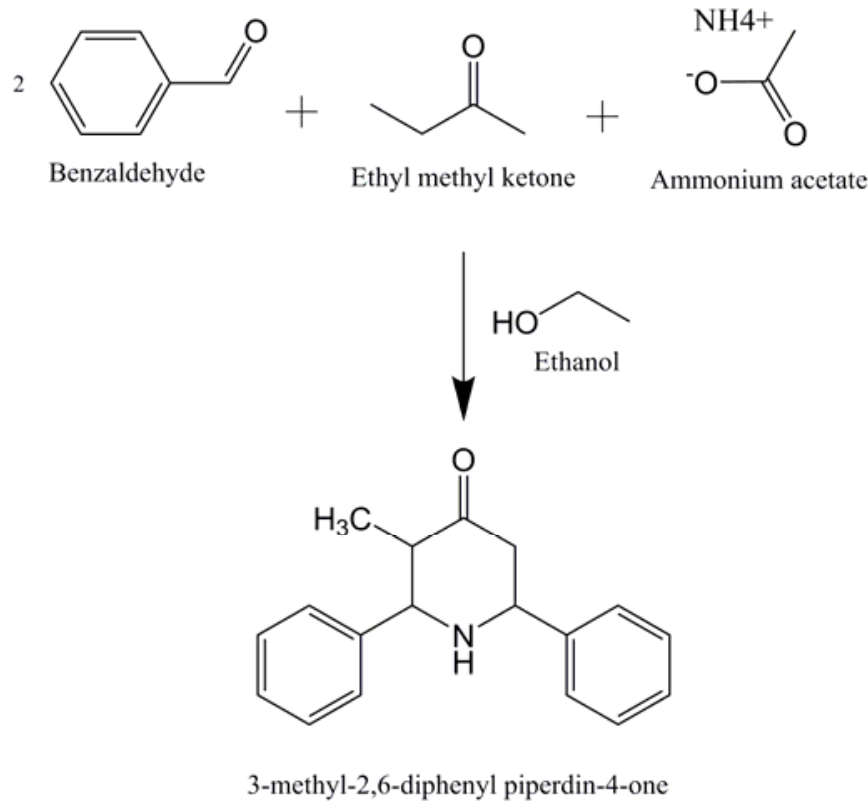

Figure 2. Synthesis of 3-methyl-2,6-diphenylpiperidin-4-one

\section{IR Spectra for ligands and complexes}

The infrared spectra for ligands $\left(\mathrm{L}_{1}-\mathrm{L}_{9}\right)$, shows an absorption band lies around $1700 \mathrm{~cm}^{-1}$ and $3300 \mathrm{~cm}^{-1} \mathrm{C}=\mathrm{O}$ groups and $\mathrm{NH}$ groups. In Palladium complexes $\left(\mathrm{C}_{1}-\mathrm{C}_{9}\right)$, it is shifted corresponding of the ligands are recorded in Table 1.

Table 1. IR spectral data for ligands and complexes

\begin{tabular}{ccccccccc}
\hline \multirow{2}{*}{ Complex } & \multicolumn{7}{c}{$\mathrm{IR}$} \\
\cline { 2 - 9 } & $v(\mathrm{C}=\mathrm{O})^{\mathrm{a}}$ & $v(\mathrm{C}=\mathrm{O})^{\mathrm{b}}$ & $v(\mathrm{~N}-\mathrm{H})$ & $v(\mathrm{O}-\mathrm{H})$ & $v(\mathrm{HOH})$ & $v(\mathrm{Ar}-\mathrm{H})$ & $v(\mathrm{M}-\mathrm{N})$ & $v(\mathrm{M}-\mathrm{O})$ \\
\hline $\mathrm{C}_{1}$ & 1710 & 1716 & 2928 & $3200-3700$ & 1633 & 802 & 698 & 435 \\
$\mathrm{C}_{2}$ & 1712 & 1716 & 2924 & $3200-3700$ & 1624 & 868 & 551 & 513 \\
$\mathrm{C}_{3}$ & 1713 & 1716 & 2924 & $3200-3700$ & 1625 & 867 & 547 & 460 \\
$\mathrm{C}_{4}$ & 1697 & 1716 & 2928 & $3200-3700$ & 1631 & 810 & 516 & 451 \\
$\mathrm{C}_{5}$ & 1712 & 1716 & 3207 & $3200-3700$ & 1620 & 817 & 516 & 455 \\
$\mathrm{C}_{6}$ & 1720 & 1722 & 2926 & $3200-3700$ & 1618 & 812 & 514 & 422 \\
$\mathrm{C}_{7}$ & 1705 & 1716 & 2928 & $3200-3700$ & 1621 & 829 & 536 & 424 \\
$\mathrm{C}_{8}$ & 1701 & 1718 & 3209 & $3200-3700$ & 1612 & 804 & 540 & 401 \\
$\mathrm{C}_{9}$ & 1707 & 1714 & 3089 & $3200-3700$ & 1612 & 831 & 538 & 418 \\
\hline
\end{tabular}

$v(C=O)^{a}$-for ligands; $v(C=O)^{b}$ - for complexes

\section{${ }^{1} H$-NMR spectra}

The nine complexes $\left(\mathrm{C}_{1-} \mathrm{C}_{9}\right)$ were characterized by ${ }^{1} \mathrm{H}$ NMR spectroscopy. The ${ }^{1} \mathrm{H}$ NMR spectral data shows a singlet at $\delta 2.5 \mathrm{ppm}$ to $\mathrm{NH}$ protons are given in Table 2 . 
Table 2. ${ }^{1} \mathrm{H}$ NMR spectral data for complexes

\begin{tabular}{cccccccccc}
\hline \multirow{2}{*}{ NMR } & \multicolumn{8}{c}{ Complex } \\
\cline { 2 - 9 } & $\mathrm{C}_{1}$ & $\mathrm{C}_{2}$ & $\mathrm{C}_{3}$ & $\mathrm{C}_{4}$ & $\mathrm{C}_{5}$ & $\mathrm{C}_{6}$ & $\mathrm{C}_{7}$ & $\mathrm{C}_{8}$ & $\mathrm{C}_{9}$ \\
\hline $\mathrm{H}(2)(\mathrm{s}, 1 \mathrm{H})$ & 3.327 & 3.332 & 3.344 & 3.344 & 3.322 & 3.502 & 3.359 & 3.363 & 3.336 \\
$\mathrm{H}(3)$ and & 2.148 & 2.508 & 2.509 & 3.790 & 3.402 & 2.740 & 3.781 & 3.608 & 3.796 \\
$\mathrm{H}(5)(\mathrm{m}, 3 \mathrm{H})$ & & & & & & & & & \\
$\mathrm{H}(6)(\mathrm{s}, 1 \mathrm{H})$ & 3.584 & 3.514 & 4.728 & 4.145 & 4.412 & 3.344 & 5.511 & 5.489 & 5.502 \\
$\mathrm{C}(3)-\mathrm{R}(\mathrm{d}, 3 \mathrm{H})$ & 0.668 & 0.664 & 0.717 & 0.770 & 0.629 & 0.731 & 0.642 & 0.624 & 0.720 \\
$\mathrm{NH}(\mathrm{s}, 1 \mathrm{H})$ & 2.501 & 2.306 & 2.509 & 2.506 & 2.502 & 2.505 & 2.505 & 2.504 & 2.504 \\
Aromatic & 7.735 & 7.394 & 7.426 & 6.437 & 7.268 & 7.426 & 7.125 & 6.910 & 7.452 \\
protons & - & - & - & - & - & - & - & - & - \\
& 7.865 & 7.540 & 7.516 & 7.611 & 9.994 & 10.544 & 7.750 & 7.375 & 10.790 \\
\hline
\end{tabular}

${ }^{13}$ C NMR spectra

${ }^{13} \mathrm{C}$ NMR spectrashows a peak $\delta 204$ ppm for $\mathrm{C}=\mathrm{O}$ group in $\mathrm{C}_{4}$ carbon by shifting the position on complexion with precursor Table 3 .

Table 3. ${ }^{13} \mathrm{C}$ NMR spectral data for complexes

\begin{tabular}{cccccccccc}
\hline \multirow{2}{*}{ NMR } & \multicolumn{8}{c}{ Complex } \\
\cline { 2 - 11 } & $\mathbf{C}_{\mathbf{1}}$ & $\mathbf{C}_{\mathbf{2}}$ & $\mathbf{C}_{\mathbf{3}}$ & $\mathbf{C}_{\mathbf{4}}$ & $\mathbf{C}_{\mathbf{5}}$ & $\mathbf{C}_{\mathbf{6}}$ & $\mathbf{C}_{\mathbf{7}}$ & $\mathbf{C}_{\mathbf{8}}$ & $\mathbf{C}_{\mathbf{9}}$ \\
\hline $\mathrm{C}(2)$ & 70.417 & 70.281 & 68.172 & 60.321 & 70.185 & 63.416 & 67.553 & 67.849 & 61.953 \\
$\mathrm{C}(3)$ & 64.126 & 64.561 & 64.068 & 53.901 & 64.649 & 59.560 & 60.508 & 55.460 & 55.563 \\
$\mathrm{C}(4)$ & 204.320 & 209.018 & 206.940 & 208.270 & 209.159 & 203.821 & 209.673 & 211.590 & 210.123 \\
$\mathrm{C}(5)$ & 56.140 & 48.328 & 59.432 & 49.449 & 48.932 & 45.266 & 55.769 & 51.421 & 51.466 \\
$\mathrm{C}(6)$ & 46.266 & 40.264 & 40.187 & 40.190 & 40.280 & 40.265 & 40.184 & 40.196 & 40.276 \\
$\mathrm{C}(3)-\mathrm{R}$ & 11.180 & 11.167 & 11.134 & 10.806 & 11.180 & 11.114 & 11.837 & 11.119 & 12.076 \\
Aromatic & 127.430 & 128.878 & 128.722 & 106.065 & 127.248 & 128.828 & 110.052 & 129.151 & 128.256 \\
carbons & - & - & - & - & - & - & - & - & - \\
& 128.880 & 138.322 & 139.218 & 155.696 & 139.151 & 135.722 & 128.877 & 135.080 & 130.032 \\
\hline
\end{tabular}

\section{Conclusion}

From the above results and discussion for a series of substituted piperdin-4-one ligands $\left(\mathrm{L}_{1}-\mathrm{L}_{9}\right)$ and their complexes $\left(\mathrm{C}_{1}-\mathrm{C}_{9}\right)$ were synthesized and characterized successfully.

\section{Acknowledgment}

The author wishes to express their thanks to Principal, the Head and Staff of Chemistry Department, PSG College of Technology for providing laboratory facility.

\section{References}

1. Garrafo H M, Caceres J, Daly J W, Spande T F, Andriamaharavo N R and Andriantsiferana M, J Nat Prod., 1993, 56(7), 1016-1038; DOI:10.1021/np50097a005

2. Angle S R and Breitenbucher J G. Studies in Natural Products Chemistry, Elsevier 1995, 16, 453-502.

3. Padmavathi V, Ramana Reddy T V, Audisesha Reddy K, Padmaja A and Bhaskar Reddy A, Indian J Chem., 2005, 44B, 2527-2531.

4. $\quad$ Kartritzky A R and Fan W Q, J Org Chem., 1990, 55(10), 3205-3209; DOI:10.1021/jo00297a041 
5. Ramalingam C, Balasubramanian S, Kabilan S and Vasudevan M, Eur J Med Chem., 2004, 39(6), 527-533; DOI:10.1016/j.ejmech.2004.02.005

6. Ramalingam C, Balasubramanian S and Kabilan S, Synth Commun., 2003, 33(9), 1443-1448; DOI:10.1081/SCC-120018753

7. Ramalingam C, Balasubramanian S, Kabilan S and Vasudevan M, Med Chem Res., 2003, 12, 26-40.

8. Ramalingam C, Balasubramanian S, Kabilan S and Vasudevan M, Med Chem Res., 2003, 12, 41-55.

9. Ramalingam C, Balasubramanian S and Kabilan S, Synth Commun., 2004, 34(36), 1105-1116; DOI: $10.1081 /$ SCC-120028643

10. Ramalingam C, Balasubramanian Sm and Kabilan S, Synth Commun., 2003, 33(17), 2979-2984; DOI:10.1081/SCC-120022470

11. Manimekalai A, Jayabharathi J, Porselvi V M and Prabha N, Indian J Chem., 2007, 46B, 681-689.

12. Thennarasu S and Perumal P T, Molecules, 2002, 7(6), 487-493.

13. Noller C R and Baliah V, J Am Chem Soc., 1948, 70(11), 3853-3855;

DOI:10.1021/ja01191a092 